\title{
Topology Optimization Under Uncertainty by Using the New Collocation Method
}

\author{
Seyyed Ali Latifi Rostami ${ }^{1}$, Ali Ghoddosian ${ }^{1 *}$ \\ ${ }^{1}$ Department of Mechanical Engineering, \\ Faculty of Engineering \\ Semnan University, P.O.B: 35131-19111, Semnan, Iran \\ * Corresponding author, e-mail: aghoddosian@semnan.ac.ir
}

Received: 01 September 2018, Accepted: 03 January 2019, Published online: 04 February 2019

\begin{abstract}
In this paper, a robust topology optimization method presents that insensitive to the uncertainty in geometry. Geometric uncertainty can be introduced in the manufacturing variability. This uncertainty can be modeled as a random field. A memory-less transformation of random fields used to random variation modeling. The Adaptive Sparse Grid Collocation (ASGC) method combined with the geometry uncertainty models provides robust designs by utilizing already developed deterministic solvers. The proposed algorithm provides a computationally cheap alternative to previously introduced stochastic optimization methods based on Monte Carlo sampling by using the adaptive sparse grid method. The method is demonstrated in the design of a minimum compliance Messerschmitt-Bölkow-Blohm (MBB) and cantilever beam as benchmark problems.
\end{abstract}

Keywords

topology optimization, geometric uncertainty, sparse grid, collocation method

\section{Introduction}

Structural optimization, an essential element of engineering design structures to improve performance and reduce costs. The main components of structural optimization in general, are divided into three levels, namely, optimizing the size, shape, and topology. The purpose of the classic topology optimization is to obtain an optimal distribution of material or structural design parameters in a range of nominal material properties, geometry and loading conditions.

SIMP and ESO methods are known as two ways very popular in topology optimization and many articles by using these methods have been presented. It is essential to note these methods are formulated based on elemental design variables. Although the use of these variables in the formulation of material distribution was seen natural, cause some problems in topology optimization can also be expressed as follows: (1) checkerboard patterns, (2) mesh dependency, (3) local optimum.

To overcome these problems, various methods have been proposed. For example, Matsui and Terada [1] have presented the CAMD model (a continuous approximation of material distribution) that combined with homogeneous topology optimization methods. Kang and Wang [2] have proposed another approach in this area based on a Shepard function interpolation with higher-order elements to maintain the physical meaning of variable density is used in structural topology optimization.

Traditionally, the structural topology optimization is done in a certain manner and known in the design as a deterministic topology optimization (DET), regardless of determining the various sources of uncertainty [3]. However, designs found by deterministic approaches are often sensitive to variations of the system and operating parameters, and therefore of limited value in practice. To mitigate this issue, safety factors are traditionally introduced into the formulation of the design optimization problem, often leading to unknowingly unsafe or overly conservative designs. Thus, a strong need exists to consider the effect of uncertainty on optimum structural topology design.

Ben-Tal et al. [4] presented an approach based on semi-definite programming for strong structural truss topology optimization according to the requirements uncertain load. Guest and Igusa [5] considered topology optimization with the uncertainty of the size and location of applied loads and with small uncertainties in the 
structural nodes. In their research to resolve the problem of loading uncertainty, the method of weighted average multiple load patterns has been developed.

Kogiso et al. [6] considered uncertainty in the direction of the driving force and examined the optimization of the complaint mechanisms. Variations were studied based on the sensitivity of the evaluated variance by using the first derivative. Dunning et al. [7], Cherkaev [8], Logo [9] and Logo et al. [10], Zhao et al.[11], Zhao et al. [12], were also studied robust topology optimization under uncertainty loading.

Chen et al [13] by using the level set method proposed a robust design method for structures. They considered a robust design for minimum compliance and complaint mechanisms. The robust shape and topology optimization (RSTO) by taking into account uncertainties in loading and material properties have been studied in their work. The novel aspect of their approach is the use of the spectral stochastic finite element method (SSFEM) to the model random field. Important work has very recently been done on the robust shape and topology optimization of two-dimensional structures (Tootkaboni et al. [14]) for mass minimization, using a polynomial chaos approach.

Modeling geometric uncertainty in topology and shape optimization has been the focus of some researchers using both level set and density-based methods [13, 15, 16]. In density based topology optimization these uncertainties that are attributed to manufacturing tolerances are commonly modeled via the Heaviside thresholding approach [17-20]. In earlier studies [20, 21], due to over and under etching, the authors consider manufacturing variances as a uniform threshold field. Their approach uses a min-max design, a formulation that considers the nominal, overetched and under-etched scenarios. These studies have since been extended and they are considered non-uniform variations. In non-uniform consideration, the threshold is represented by a random field that is parameterized by a Karhunen Loeve Expansion (KLE).

Chen and Chen [15] by using a level-set approach extended topology optimization in the geometric uncertainties field. They have presented an RDO for topology optimization of structures using level-sets. They used HamiltonJacobi equations for modeling a stochastic velocity field. Then, random geometry variations are modeled through the velocity field. The stochastic moments of the design criteria are evaluated using an efficient quadrature rule. After solving analytically derived adjoint equations, the shape sensitivities for the uncertain geometry are computed.
Lazarov et al. [19] used the stochastic perturbation method to model geometric uncertainty. The main assumption is that the random variability of the system parameters, the inputs, and the solution is small. The method provides a computationally cheap alternative to $\mathrm{MC}$, however, the requirement for small variability imposes restrictions on the general applicability.

Keshavarzzadeh et al. [22] present a systematic approach to topology optimization under uncertainty in loading and in geometry. They used that integrates non-intrusive polynomial chaos expansion with design sensitivity analysis for reliability-based and robust topology optimization. By using of thresholding technique, the manufacturing variability is modeled. In this method, the threshold field is demonstrated as a reduced dimensional random field. Response metrics such as compliance and volume are characterized as polynomial chaos expansions of the underlying uncertain parameters, thus allowing accurate and efficient estimation of statistical moments, failure probabilities and their sensitivities.

This paper organized as follows. First the deterministic (DET) optimization problem and then the Stochastic optimization approach for obtaining robust designs are presented. In the next section, modeling of geometric uncertainty is discussed. Next, Adaptive Sparse Grid Collocation methods and its features are introduced. The optimization algorithm is presented in the next section and finally, its applicability in topology optimization of robust minimum compliance is demonstrated.

\section{Problem definition}

One of the issues that are commonly discussed in topology optimization is the minimum compliance optimization. The formulation of this field is given as

$$
\begin{aligned}
& \underset{\rho}{\operatorname{Min}}: f(\rho)=\mathbf{l}^{T} \mathbf{u} \\
& \text { s.t.: Ku }=\mathbf{f} \\
& : \boldsymbol{V}(\rho)=\sum_{i=1}^{N_{e}} \tilde{\bar{\rho}}_{i} V_{i} \leq V^{*} \\
& : 0 \leq \rho \leq 1 \quad \forall i \in N_{e},
\end{aligned}
$$

where $\mathbf{K}$ is the stiffness matrix obtained by finite element discretization, $\mathbf{u}$ and $\mathbf{f}$ is the solution and the input vectors for the system $N_{e}$ are the set of all elements and $\tilde{\bar{\rho}}_{i}$ are the physical density associated with. $V_{i}$ is a volume of element $i, V$ is the total volume of the design domain and $V^{*}$ is the fraction of the total volume which can be occupied with the material. The individual elements contributions to the 
tangent matrix $\mathbf{K}$ are calculated as $\mathbf{K}_{i}=E_{i} \mathbf{K}_{0}$ where $\mathbf{K}_{0}$ is the element stiffness matrix for unit stiffness and $E_{i}$ is the material stiffness obtained by using the so-called solid isotropic material interpolation with penalization (SIMP) given as

$E_{i}=E_{\text {min }}+\tilde{\bar{\rho}}_{i}^{p}\left(E_{0}-E_{\text {min }}\right)$,

where $E_{0}$ indicates the stiffness of places that occupied with the material, $p$ is the penalization factor and $\tilde{\bar{\rho}}_{i}$ is the physical density of element $i$. The vector $\mathbf{l}$ in Eq. (1) will have different values for different problems. In the test case that discussed minimum compliance design, $\mathbf{I}=\mathbf{f}$.

Problems governed by the mechanical stiffness suffer from numerical instabilities, such as checkerboard patterns and mesh dependency solution. Density filtering makes it possible to achieve independent designs of the mesh.

Here the mesh independent density filtering (Bruns and Tortorelli [23]) is used as a basis to ensure the existence of solutions. The basic idea is to determine the physical element density to be a weighted average of the neighboring design variables, where the neighborhood is defined by a circle in $2 \mathrm{D}$ or sphere in $3 \mathrm{D}$ with the specified radius. Applying regularization to the original problem causes gray areas with a moderate density of 0 to 1 .

In the following, the filtered density is denoted with $\tilde{\rho}_{i}$ and physical density with $\tilde{\bar{\rho}}_{i}$. The filtered density for $i$-th element is calculated as

$\tilde{\rho}_{i}=\frac{\sum_{j \in N_{e, i}} w\left(\mathbf{x}_{j}\right) v_{j} \rho_{j}}{\sum_{j \in N_{e, i}} w\left(\boldsymbol{x}_{j}\right) v_{j}}$,

where the neighborhood set of elements, locating within the filter domain for the element $i$ am represented by, the weighting function $w(\mathbf{x})$ is defined as

$w\left(\mathbf{x}_{j}\right)=R-\left|\mathbf{x}_{j}-\mathbf{x}_{i}\right| \cdot$

In the above relation, $R$ the specified filter radius and, $\mathbf{x}_{i}$ and $\mathbf{x}_{j}$ are central coordinates of the design elements $i$ and $j$ respectively. The sensitivity of filtered density $\tilde{\rho}_{i}$ with respect to the design variables is calculated as

$\frac{\partial \tilde{\rho}_{i}}{\partial \rho_{i}}=\frac{w\left(\mathbf{x}_{j}\right) v_{j}}{\sum_{j \in N_{e, i}} w\left(\mathbf{x}_{j}\right) v_{j}}$.

However, if the physical density is shown by the filtered density, the design of the gray areas will be formed, which will be difficult to interpret. Projection schemes should be used to convert these gray areas to white and black areas. In this research, a Heaviside projection procedure is used for projecting these gray areas. In this method firstly a threshold $\eta$ is defined and then all values below threshold are projected for 0 and above the threshold to 1 . As the Heaviside function is not differentiable, it is approximated by a smooth approximation controlled by relaxation parameter $\beta$. A Heaviside projection utilized here is given as

$\tilde{\bar{\rho}}_{i}=\frac{\tanh (\beta \eta)+\tanh \left(\beta\left(\tilde{\rho}_{i}-\eta\right)\right.}{\tanh (\beta \eta)+\tanh (\beta(1-\eta)}$.

The derivative of the physical density $\tilde{\bar{\rho}}_{i}$ with respect to the filtered density $\tilde{\rho}_{i}$ is calculated as

$$
\frac{\partial \tilde{\bar{\rho}}_{i}}{\partial \tilde{\rho}_{i}}=\frac{\beta\left(\operatorname{sech}\left(\beta\left(\tilde{\rho}_{i}-\eta\right)\right)^{2}\right.}{\tanh (\beta \eta)+\tanh (\beta(1-\eta)} .
$$

The physical density $\tilde{\bar{\rho}}_{i}$ is a function of the filtered density $\tilde{\rho}_{i}$ and the sensitivities of the objective function in Eq. (1) with respect to the original design variables are calculated as follows

$$
\frac{\partial f}{\partial \rho_{j}}=\sum_{i \in N_{e, j}} \frac{\partial f}{\partial \tilde{\bar{\rho}}_{i}} \frac{\partial \tilde{\bar{\rho}}_{i}}{\partial \tilde{\rho}_{i}} \frac{\partial \tilde{\rho}_{i}}{\partial \rho_{j}} .
$$

\section{Stochastic optimization}

When a stochastic system is discussed, its properties such as excitations, material or manufacturing errors will have a random nature (depending on the type of problem). Therefore the response $\mathbf{u}$ becomes a stochastic field and the objective in Eq.(1) becomes a random variable. The stochastic compliance objective function in robust form is commonly determined by using of the mean and standard deviation of the compliance in the form of the weighted sum.

$$
\begin{aligned}
& \operatorname{Min}: f(\rho)=E[C]+\kappa \sqrt{\operatorname{Var}[C]} \\
& =E\left[\mathbf{l}^{\mathrm{T}} \mathbf{u}\right]+\kappa \sqrt{\operatorname{Var}\left[\mathbf{I}^{\mathrm{T}} \mathbf{u}\right]} \\
& \text { s.t.: Ku}=\mathbf{f} \\
& : V(\rho) \leq V^{*} \\
& : \mathbf{0} \leq \boldsymbol{\rho} \leq \mathbf{1} \quad \forall \boldsymbol{i} \in \boldsymbol{N}_{\boldsymbol{e}}
\end{aligned}
$$

where $E[C]$ is the expected value of the compliance, the variance of the compliance is shown with $\operatorname{Var}[C]$ and $\kappa$ represented the weighting coefficient that chosen by the user. In deterministic loading condition, the mean value (Expectancy) and the variance of the compliance of are given by

$$
E[C]=E\left[\mathbf{f}^{\mathrm{T}} \mathbf{u}\right]=\mathbf{f}_{0}^{T} E[\mathbf{u}],
$$




$$
\operatorname{Var}[C]=\operatorname{Var}\left[\mathbf{f}^{T} \mathbf{u}\right] .
$$

The sensitivities of the objective function with respect to the design variables $\boldsymbol{\rho}$ are found by using the adjoint method as follows

$$
\frac{\partial f}{\partial \boldsymbol{\rho}}=\frac{\partial E[C]}{\partial \boldsymbol{\rho}}+\kappa \frac{\partial(\sqrt{\operatorname{Var}[C]})}{\partial \boldsymbol{\rho}} .
$$

In the following sections first, the representation of the uncertainties as a stochastic field is discussed and then the solution of the stochastic state problem by using the Probabilistic collocation method is presented in more details.

\section{Manufacturing uncertainty via random field}

We now consider uncertainty geometry in the above optimization problem. As mentioned above, geometric uncertainties are modeled by introducing randomness to the threshold parameter $\eta$. This approach is model uncertainty in structures that are fabricated via etching. The etching process causes errors in the form of over- or under-etching which produces structures that are either thinner or thicker than intended. To model this uncertainty the Heaviside threshold is applied. The etching can cause, a more realistic assumption, a non-uniform variation of errors in the design domain. In the present paper this variation represents by replacing the random variable $\eta$ with the random field such that:

$$
\eta(x, \xi)=\alpha_{1} \hat{Z}(x, \xi)+\alpha_{2},
$$

where $\hat{Z}(x, \xi) \in[0,1]$ is a random field, $\alpha_{1}$ and $\alpha_{2}$ control the mean and range of the process $\eta$ such that $\eta(x, \xi) \in[0,1]$.

We use a truncated KLE to model the random field $\eta$. The KLE provides a mapping from a relatively small number of independent random variables to the types of random fields that are common in many physical processes. Random field $Z$ as a result of KLE, however, does not have the optimal range for topology optimization which we need to $\eta$ values between 0 and 1 . As such, an inverse transform sampling is applied to $Z$ to ensure the desirable variation of random variables $\hat{Z}$ and thus $\eta$.

To obtain the KLE expansion for the random field $Z$ its correlation function is assumed to be of the squared exponential form i.e.

$$
R_{x x^{\prime}}=\exp \left(-\frac{d^{2}}{2 l_{c}^{2}}\right),
$$

where $d=|x-\dot{x}|$ is the Euclidean distance between locations $x$ and $\dot{x}, l_{c}$ is the correlation length. We discretize our space so $x$ and $x^{\prime}$ are the finite element centroids to obtain the correlation matrix $R$. The $n$ eigenvalue-eigenvector pairs $\left(\lambda_{i}, \gamma_{i}\right)$ of the correlation matrix are subsequently used to generate the KL decomposition of a zero mean process as

$$
Z(x, \xi)=\sum_{i=1}^{n} \sqrt{\lambda_{i}} \gamma_{i}(x) \varphi_{i}(\xi) \approx \sum_{i=1}^{n_{\text {mode }}} \sqrt{\lambda_{i}} \gamma_{i}(x) \varphi_{i}(\xi),
$$

where $\varphi_{i}$ are random variables and $\gamma_{i}(x)$ is interpolated from the eigenvector $\gamma_{i}$ as piecewise uniform over the elements. The expansion is truncated to the first $n_{\text {mode }}<n$ modes for the purpose of dimensionality reduction. It is noted that the number of modes $n_{\text {mode }}$ is dependent on the correlation length $l_{c}$ and the type of the correlation function.

Ultimately the random variables $\varphi_{i}$ are used to generate the random field $Z$ via Eq. (15). To that end, we assign uniform random variables to each $\varphi_{i}$ with zero mean and unit variance, i.e. $\varphi_{i}(\xi)=\xi_{i}=U[-\sqrt{3}, \sqrt{3}]$. This zero mean and unit variance choice ensures that the same correlation matrix $\mathbf{R}$ can be reconstructed from the random field $Z$ of Eq. (15).

Unfortunately, realizations of the $\operatorname{KLE} Z(x, \xi) \notin[0,1]$ and this is noteworthy. Indeed, our geometric uncertainty is introduced by randomly varying the threshold parameter $\eta$ and we require $\eta(x, \xi) \in[0,1]$. To generate the random field $\hat{Z}$ of Equation (13) such that $\hat{Z}(x, \xi) \in[0,1]$ we use the fact that the Cumulative Density Function (CDF) of any continuous random variable, e.g. $Z(x, \xi)$ is a uniform random variable, ranging from 0 to 1 i.e. $U[0,1]$; this is ideal for modeling thresholds. In other words, for every realization of $Z(x, \xi)$ there is a unique $\mathrm{CDF}$ transformed value that belongs to the range $[0,1]$. The ensemble of these $\mathrm{CDF}$ transformed values has a uniform distribution from which the transformed process $\hat{Z}$ is defined such that

$$
Z(x, \xi)=C D F(\hat{Z}(x, \xi)) .
$$

\section{Adaptive sparse grid collocation methods}

\subsection{Sparse grid}

The sparse grid method was introduced by Smolyak is a most popular method that very useful in multidimensional quadrature and interpolation. The sparse tensor product is the basis of this method. In following this method construction is represented. Suppose that $Q_{l}^{(1)} f$ a family of quadrature rules and will have: 
$\Delta_{l}^{(1)} f \equiv\left(Q_{l}^{(1)}-Q_{l-1}^{(1)}\right) f$

$Q_{0}^{(1)} f \equiv 0$.

Note that $\Delta_{l}^{(1)} f$ is also a quadrature rule. For nested formulas, $\Delta_{l}^{(1)} f$ contains the set of nodes of $Q_{l}^{(1)} f$ with weights equal to the difference of weights between level $l$ and $l-1$.

By introducing the multi-index $l=\left(l_{1}, \ldots, l_{N}\right) \in N^{N}$ can construct the sparse cubature, and define

$|\mathbf{I}| \equiv \sum_{i=}^{N} l_{i}$

At level $l$ this multi-index is used and then the sparse cubature formula is represented as

$$
Q_{l}^{(N)} f \equiv \sum_{|| \leq l+N-1}\left(\Delta_{l_{1}}^{(1)} \otimes \ldots \otimes \Delta_{l_{1}}^{(N)}\right) f,
$$

where multi-index expressed of the support nodes is $|\mathbf{I}|$, $\left(|\mathbf{I}|=1_{1}+\ldots+1_{d}\right)$. The dimension of function $f$ is shown by $N$. By using a recursive manner the interpolant expressed as follows:

$$
Q_{l}^{(d)} f=\sum_{l+1 \leq 1 \mid 1 \leq l+d}(-1)^{l+d-|\mathbf{k}|}\left(\begin{array}{c}
d-1 \\
l+d-|\mathbf{k}|
\end{array}\right)\left(Q_{k_{1}}^{(1)} \otimes \ldots \otimes Q_{k_{d}}^{(1)}\right) .
$$

The weight $w_{i}$ corresponding to the $i$ th collocation point $\xi_{i}$ is defined with by the Smolyak algorithm,

$w_{i}=(-1)^{l+d-\mathbf{k} \mid}\left(\begin{array}{c}d-1 \\ l+d-|\mathbf{k}|\end{array}\right)\left(w_{k_{1}}^{i_{1}} \otimes \ldots \otimes w_{k_{d}}^{i_{d}}\right)$.

Then, the mean and standard deviation of the objective of the sparse grid method can be computed by

$$
\begin{aligned}
& E[f]=\sum_{k} w_{k} f\left(\xi_{k}\right) \\
& \operatorname{Var}[f]=E[f]-(E[f])^{2} .
\end{aligned}
$$

\subsection{Adaptive sparse grid}

The conventional sparse grid collocation treats all dimensions equally. In most physical problems that one deals with, there usually exists some structure (additive, nearly-additive, anisotropic, discontinuous) that can be taken advantage of to reduce the number of function evaluations. However, the specific kind of structure that the particular solution exhibits are not known a priori. Thus, one must construct an approach that automatically detects which dimensions require more nodal points or where the discontinuity happens. The basic proposition of an adaptive sparse grid collocation method is to assess the stochastic dimensions differently, according to the error of interpolation in that dimension.

One way to perform adaptation and refinement is on the level of the hierarchical subspace. This leads to the so-called dimension-adaptive (anisotropic) sparse grids. This approach detects important dimensions and places all the collocation points along the important dimension.

Sequential construction of the multi-index set is the basis of the adaptation, which is progressively enriched starting from $I=\{\mathbf{1}=(1, \ldots, 1)\}$. At first, the notion of the admissible multi-index set will be introduced. Suppose that an index set $I$ is admissible if for each multi-index $\mathbf{I} \in I$ we have $\mathbf{I}-$ $e_{j} \in I$, for $1 \leq j \leq N, l_{j}>1$, where $e_{j}$ is $j$-th unit vector $\left(\left(e_{j}\right)\right.$ $i=\delta_{i j}$ ). This condition means that in all directions any element of I has a predecessor. Based on this feature the telescope sum expansion when defining a sparse grid curvature is validated in terms of differing rules. In Fig.1, for the case of $N=2$ admissible and non-admissible multi-index sets are shown. The adaptive sparse grid method requires that the progressive enrichment of the multi-index set maintains the admissibility. In addition, the enrichment should reduce the integration error in the most efficient way. To this end, which multi-index should be added to $I$ is determined by using an indicator. The indicator error denoted as $g_{k}$ which associated to a multi-index $\mathbf{k}$. The $\mathrm{g}_{\mathrm{k}}$ combines information from the associated difference term, $\Delta_{\mathrm{k}}^{(N)} f$, with the computational complexity involved in its estimation. The latter is measured by $n_{k}$ defined as the number of cubature nodes in the evaluation of $\Delta_{\mathrm{k}}^{(N)} f$. A convenient form for $\mathrm{g}_{\mathrm{k}}$ is:

$\mathrm{g}_{\mathrm{k}} \equiv \max \left\{\alpha\left|\frac{\Delta_{\mathrm{k}}^{(N)} f}{\Delta_{1}^{(N)} f}\right|,(1-\alpha) \frac{\mathrm{n}_{1}}{\mathrm{n}_{\mathrm{k}}}\right\}$,

where $0 \leq \alpha \leq 1$ weight the difference contribution and computational cost.

The enrichment of $I$ with this indicator can proceed. Suppose that for a given step of the adaptation an admissible multi-index set $I$ have constructed, and for $\mathbf{I} \in I$ its forward neighborhood $F_{1}$ defined as
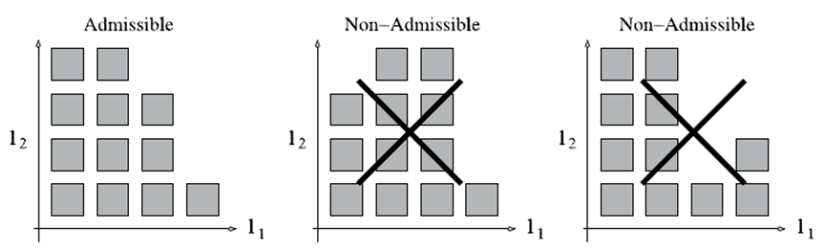

Fig. 1 Examples of admissible and non-admissible multi-index sets in two dimensions 
$F_{1} \equiv\left\{\mathbf{I}+\boldsymbol{e}_{j}, 1 \leq j \leq N\right\}$.

The next multi-index denoted $\mathbf{k}$, is to be selected based on the following conditions
$\mathbf{k} \notin I$
(a)
$\mathbf{k} \in \bigcup_{\mathbf{l} \in I} F_{\mathbf{1}}$
$I \cup\{\mathbf{k}\}$ is admissible.
(c)

Based on these conditions, (a) $\mathbf{k}$ should be a new multi-index, (b) taken in the forward neighborhood of $I$, whose inclusion leaves $I$ admissible (c). This method is effectively implemented with two subsets $O$ and $A$.

The "old" multi-indexes represented by the set $O$ which need not be tested anymore, while those who are candidates for inclusion in $I$ are in a set $A$. The set $O$ is initialized to $\{\mathbf{1}\}$ and $A$ to $F_{1}$. We then select the multi-index having the highest indicator in $A$, say $\mathbf{l}$. The multi-index $\mathbf{l}$ is added to the $O$ and removed from $A$; then $A$ is completed by the multi-indexes in the forward neighborhood $F_{1}$ that maintain $I=O \cup A$ admissible, and for the new multi-indexes in $\mathrm{A}$, the error indicators $\mathrm{g}_{\mathrm{k}} \notin F_{1}$ of are computed. The procedure is repeated when the global error indicator $\eta$,

$\eta \equiv \sum_{\mathbf{l} \in A} \mathbf{g}_{1}$

is greater than a prescribed error tolerance $\notin$.

\section{Optimization algorithm}

All figures must be embedded in the document when the paper is submitted to review. In the final version, however, we need figures in separate files.

The optimization algorithm using the Adaptive Sparse Grid Collocation methods can be written as follows:

1. Problem discretize and initialization progress were performed

2. Stochastic field discretization - KL by Eq. (15).

3. $M$ integration points and the corresponding weights using the adaptive sparse grid method Generated.

4. Optimization loop performed until the convergence criterion is satisfied:

- For $k=1 \ldots M$ computes $\mathbf{K}_{k} \mathbf{u}_{k}=\mathbf{f}_{k}$ and $\partial C_{k} / \partial \rho$.

- The mean and the variance Estimated from Eq. (23)

- The mean and variance sensitivities with respect to design variable evaluated

- The robust objective sensitivities computed from Eq. (12)

- Update $\boldsymbol{\rho}$.
The mean and the variance gradients are computed based on the gradients of the samples. For each sample, the standard adjoint sensitivity analysis is used for estimating the sensitivities of the objective $\partial C_{k} / \partial \rho$ and the constraints.

Keeping the solution vectors $\mathbf{u}_{k}$ can become very expensive in terms of memory. Their contribution to the expectation and the variance, as well as to the sensitivities, can be added during the loop through the collocation points.

\section{Numerical examples}

The presented methodology is demonstrated in the design of a 2D cantilever and an MBB beam. The results are obtained with a modulus of elasticity, $\mathrm{E}=1.0$, a penalization parameter $p=3$, and $\mathrm{E}_{\min }=10^{-4}$. All optimizations start with, and the projection parameter is doubling every 50 iterations. The final projection parameter is $\beta=10$. Control parameters in Eq. (13), have been considered as $\alpha_{1}=0.5$ and as $\alpha_{2}=0.25$ . The design variables are updated using optimality criteria (OC) method. The optimization process is terminated when the largest change in the design variables becomes smaller than $1 \%$. The topology optimization process is performed using MATLAB software [24]. The first 100 eigenvalues of the correlation matrix $\mathrm{R}$ over a uniform mesh are shown in Fig. 2, where their fast modal decay is apparent.

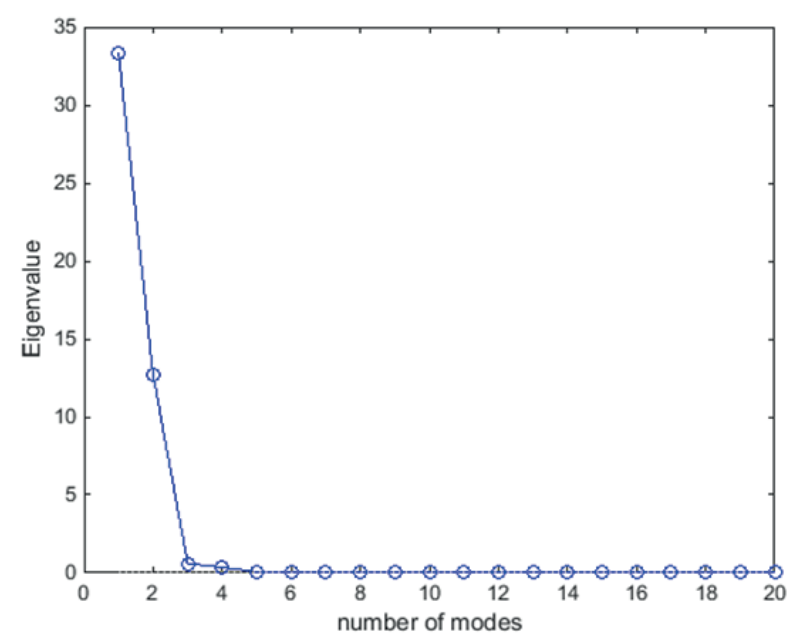

Fig. 2 Correlation eigenvalues of random field $Z$

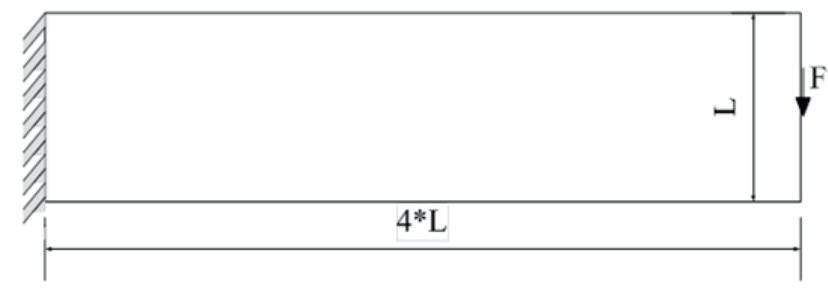

Fig. 3 Design domain and boundary condition for a cantilever beam 

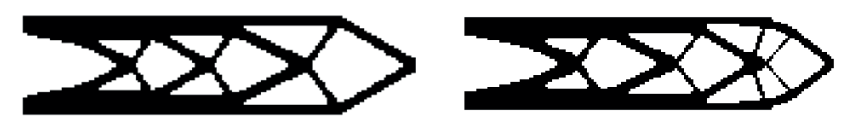

Fig. 4 Design obtained for deterministic (right) and robust design optimization (RDO)(left)
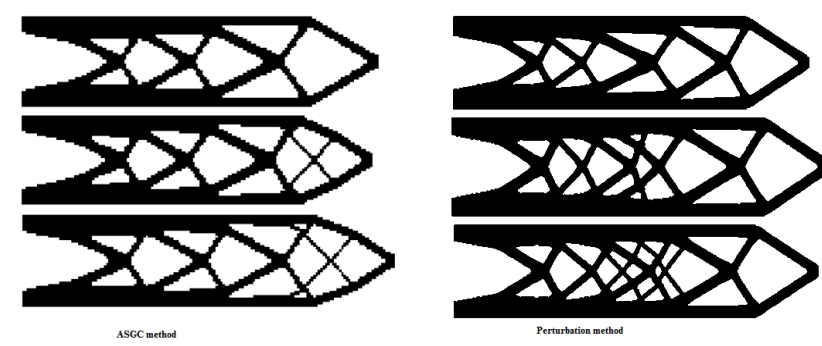

Fig. 5 Robust designs obtained with $\kappa=1$ (top), $\kappa=3, \kappa=5$ (bottom)

In practice, we truncate (KL) to first $\mathrm{N}$ terms. We used the ratio $\left(\sum_{i=1}^{n_{\text {mode }}} \lambda_{i} / \sum_{i=1}^{n} \lambda_{i}\right)$ to check the sufficiency of the number of truncated modes which indicates the first $n_{\text {mode }}$ modes to represent the random field. This measure for $n_{\text {mode }}=4$ is 0.9566 , i.e. this truncation yields a $96 \% Z$ representation which we deem sufficient.

\subsection{Robust design of a cantilever beam}

The design domain, the boundary conditions for the state problem are shown in Fig. 3. The simulations are performed with beam height $\mathrm{L}=1$. The applied force is $\mathrm{F}=0.1$. The volume fraction of the solid material is set to $50 \%$ of the total volume.

Optimized designs for a single uniformly distributed threshold $\eta \in \cup[0.3,0.7]$ are shown in Fig.4. The designs do not possess features comparable with the mesh size. The designs are obtained with deterministic and RDO are shown in Fig 4.

Increasing the weight of the standard deviation in the objective results in not be critical for small $\kappa$, as its contribution to the robust objective Eq. (9) is small. The results of the increase in $\kappa$ are denoted in Table 2. for $\eta \in \cup[0.3,0.7]$.

Increasing the weight $\kappa$ in the objective given in Eq. (9), decreases the mean compliance and decreases its variation. Therefore, the mechanism response becomes more robust with respect to variations in the geometric variation.

To validate the present method, a comparison has been done between this method with Perturbation method (Lazarov work). The results of this comparison in are denoted in Table 3 and shown in Fig. 5. RDO results related to three designs with weight parameter $\kappa=1, \kappa=3$, and $\kappa=5$.

From the results of Table 3 can be seen that the mean compliance of structure decreased with increasing in $\kappa$. Therefore, these methods have the same response. But, from
Table 1 Minimum Compliance of deterministic and robust design

\begin{tabular}{ll}
\hline Compliance & \\
\hline 3.7354 & Deterministic. \\
3.6157 & $\mathrm{RDO}(\mathrm{K}=1)$ \\
\hline
\end{tabular}

Table 2 The effect of increasing in $\kappa$ statistical moment and Compliance

\begin{tabular}{lccc}
\hline STD & Mean & Objective & \\
\hline 0.2920 & 3.2508 & 3.6157 & $\kappa=1$ \\
0.2859 & 3.1869 & 4.2591 & $\kappa=3$ \\
0.2803 & 3.1453 & 5.0499 & $\kappa=5$ \\
\hline
\end{tabular}

Table 3 Comparison of the robust design of different methods

\begin{tabular}{cccc}
\hline ASGC & Perturbation & Mont Carlo & \\
\hline 3.2508 & 3.59 & 3.58 & $\kappa=1$ \\
3.1869 & 3.67 & 3.60 & $\kappa=3$ \\
3.1453 & 3.83 & 3.62 & $\kappa=5$ \\
\hline
\end{tabular}

Table 4 The effect of increasing the threshold interval for $\kappa=3$

\begin{tabular}{lcccc}
\hline \multicolumn{2}{c}{ Present work } & \multicolumn{3}{c}{ Monte Carlo method } \\
Mean & STD & Mean & STD & Interval \\
\hline 3.1805 & 0.2843 & 3.69 & 0.42 & {$[0.1,0.9]$} \\
3.1881 & 0.2857 & 3.70 & 0.59 & {$[0.2,0.8]$} \\
3.1869 & 0.2859 & 3.70 & 0.51 & {$[0.3,0.7]$} \\
3.2465 & 0.2918 & 3.74 & 0.66 & {$[0.4,0.6]$} \\
\hline
\end{tabular}

Fig. 5 can be concluded my method has a better answer compared with the other two methods. It can be seen that when the weight parameter $\kappa$ increased, in perturbation method inside of the structure changed significantly but in the ASGC method, this changed smoothly occurred. This means that the structure becomes more robust with respect to variations in the geometric variation.

Results of increasing the threshold interval are denoted in Table 4 for $\kappa=3$. As expected, the standard deviation is decreasing with expanding the support domain of the threshold distribution this means that a larger threshold interval leads to a more robust behavior. Therefore, expanding threshold interval has a similar effect with increasing in $\kappa$.

\subsection{MBB beam}

All dimensions are in centimeters as shown by Table 1. The goal in this example is to investigate the effect of geometric uncertainty on the optimal design of the $200 \times$ $50 \mathrm{MBB}$ beam shown in Fig. 6. As explained previously, the geometric uncertainty is modeled by representing the threshold $\eta$ via a random field to model spatially varying manufacturing tolerances. 

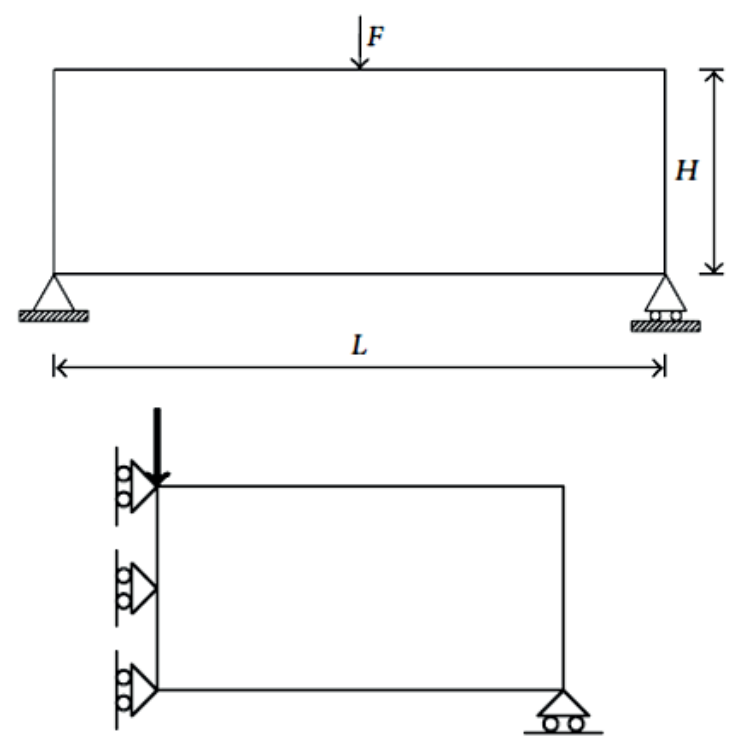

Fig. 6 The MBB-beam. Top: full design domain, bottom: half design domain with symmetry boundary conditions
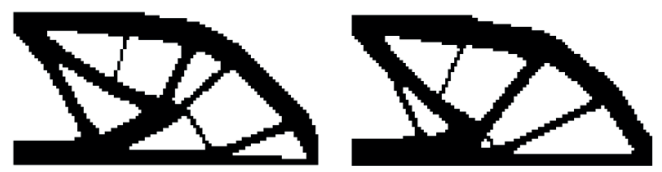

Fig. 7 Design obtained for deterministic (right) and robust design optimization (left)
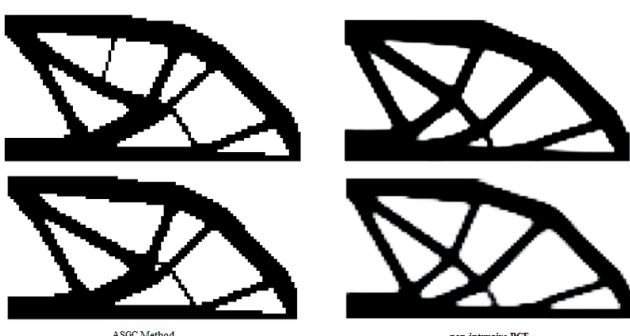

Fig. 8 Robust designs obtained with $\kappa=1$ (top), $\kappa=5$ (bottom)

The deterministic topology optimization of the MBB beam uses the symmetry of the domain whereby only half of the structure is optimized. In the stochastic analysis, however, this assumption needs to be scrutinized. We minimize the sum of the mean and standard deviations of volume subject to the RDO mean compliance constraint.

The results of these optimizations show no significant difference and it is concluded in this example that the designs obtained using the half and full domains are equivalent. As such, in the following examples, we only design on the $100 \times 50$ half-domain. A comparison of DET designs with RDO designs is shown in Fig.7. The objective functions of these designs are expressed in Table 5. Optimized designs are obtained for a uniformly distributed threshold $\eta \in \bigcup[-\sqrt{3}, \sqrt{3}]$.
Table 5 Performance comparison of Deterministic with robust designs

\begin{tabular}{lc}
\hline Compliance & Method \\
\hline 104.4844 & Deterministic \\
103.3042 & RDO $(\mathrm{K}=1)$ \\
\hline
\end{tabular}

Table 6 The effect of increasing in weight parameter $\kappa$

\begin{tabular}{lccl}
\hline Compliance & Mean. & STD & \\
\hline 103.3042 & 101.0525 & 2.2517 & $\kappa=1$ \\
108.3264 & 97.2514 & 2.2150 & $\kappa=5$ \\
\hline
\end{tabular}

Table 7 Robust design Comparison of different methods

\begin{tabular}{lcc}
\hline ASGC & non-intrusive PCE & \\
\hline 103.3042 & 120.00 & $\kappa=1$ \\
108.3264 & 120.00 & $\kappa=5$ \\
0.333 & 0.3995 & Opt Vol \\
41 & 81 & Numberof points \\
\hline
\end{tabular}

Table 8 Comparison ASGC with Monte Carlo method

\begin{tabular}{lcc}
\hline ASGC & Mont Carlo & \\
\hline 103.3042 & 105.8673 & Compliance \\
41 & $10^{5}$ & Point number \\
\hline
\end{tabular}

The effect of increasing in weight parameter $\kappa$ in optimization is denoted in Table 6. According to these results, increasing the weight $\kappa$ decreases the mean compliance and decreases its variation. Therefore, we have a more robust structure with respect to variations in the geometric variation.

In following, a comparative study has been done between the present method and a method that used in Kshavrzzadeh research. Results are shown in Fig. 8 and denoted in Table 7.

Although both methods are used of sparse grid method, according to this result can be realized the effectiveness of the multivariate hierarchical formulation. The conventional sparse grid collocation treats all dimensions equally. Thus, one must construct an approach that automatically detects which dimensions require more nodal points or where the discontinuity happens. The basic proposition of an adaptive sparse grid collocation method is to assess the stochastic dimensions differently, according to the error of interpolation in that dimension. The smaller optimum volume that expressed in Table 8 can be expected according to the results shown in Fig. 8.

To demonstrate the effectiveness of this method, we compare the compliance obtained for the RDO design with the present method and the Monte Carlo method. The results are denoted in Table 8. 
It is evident that Monte Carlo analysis with $n_{\text {samples }}=10^{5}$ is in close agreement with the non-intrusive PCE, which only requires 81 simulations, but the ASGC method by using a point number less than another method that achieves a better answer. Based on these results we can conclude this method can save time and cost.

\section{Conclusions}

Although a conclusion may review the main points of the paper, do not replicate the abstract as the conclusion. A conclusion might elaborate on the importance of the work or suggest applications and extensions.

A systematic approach for topology optimization under uncertainty is introduced that relies on the Adaptive Sparse Grid Collocation methods (ASGC) for uncertainty propagation of the cost and constraint functions. The expressions of the stochastic objective and its sensitivities are derived, and the main computational steps are presented in details. Different numerical examples for topology optimization under uncertainty are considered. The computation of the random threshold field is elucidated in the numerical examples. In these examples, geometric uncertainty is modeled by using a random threshold field that is characterized by a truncated Karhunen-Loeve expansion.

Comparing ASGC with some method such as Monte Carlo, Perturbation and sparse grid based non-intrusive PCE. It is demonstrated that the computational burden of ASGC is orders of magnitude smaller than the above-mentioned methods. For a single random variable, the presented approach is faster than optimization based on a sampling method based on Monte Carlo with $10^{5}$ realizations.

It is also shown that the optimum volume and minimum compliance obtained by using this method is smaller than other method. As well as the effect of increasing of weight parameter, $\kappa$, was discussed and showed by increasing this parameter the structure become robust and mean and standard deviation of compliance are decreased. It was shown that in comparing ASGC method and sparse grid based non-intrusive PCE, however, both methods are used of sparse grid method, but ASGC automatically detects which dimensions require more nodal points and not treats all dimensions equally.

\section{References}

[1] Matsui, K., Terada, K. "Continuous approximation of material distribution for topology optimization", International Journal for Numerical Methods in Engineering, 59(14), pp. 1925-1944, 2004. https://doi.org/10.1002/nme.945
[2] Kang, Z., Wang, Y. "Structural topology optimization based on non-local Shepard interpolation of density field", Computer Methods in Applied Mechanics and Engineering, 200(49-52), pp. 3515-3525, 2011. https://doi.org/10.1016/j.cma.2011.09.001

[3] Witteveen, J. A. S., Bijl, H. "Modeling arbitrary uncertainties using gramschmidt polynomial chaos", presented at 44th AIAA Aerospace Sciences Meeting and Exhibit, Reno, Nevada, USA, January, 9-12, 2006.

https://doi.org/10.2514/6.2006-896

[4] Ben-Tal, A., Nemirovski, A. "Robust truss topology design via semidefinite programming", SIAM Journal on Optimization, 7(4), pp. 991-1016, 1997.

https://doi.org/10.1137/S105262349529195

[5] Guest, J. K., Igusa, T. "Structural optimization under uncertain loads and nodal locations", Computer Methods in Applied Mechanics and Engineering, 198(1), pp. 116-124, 2008.

https://doi.org/10.1016/j.cma.2008.04.009

[6] Kogiso, N., Ahn, W. J., Nishiwaki, S., Izui, K., Yoshimura, M. "Robust topology optimization for compliant mechanisms considering uncertainty of applied loads", Journal of Advanced Mechanical Design, System and Manufacturing, 2(1), pp. 96-107, 2008. https://doi.org/10.1299/jamdsm.2.96

[7] Dunning, P. D., Kim, H. A., Mullineux, G. "Introducing loading uncertainty in topology optimization", AIAA Journal, 49(4), pp. 760-768, 2011

https://doi.org/10.2514/1.J050670

[8] Cherkaev, E., Cherkaev, A. "Minimax optimization problem of structural design", Computers \& Structures, 86(13-14), pp. 14261435, 2008.

https://doi.org/10.1016/j.compstruc.2007.05.026

[9] Lógó, J. "New Type of optimality criteria method in case of probabilistic loading conditions", Mechanics Based Design of Structures and Machines, 35(2), pp. 147-162, 2007. https://doi.org/10.1080/15397730701243066

[10] Lógó, J., Ghaemi, M., Rad, M. M. "Optimal topologies in case of probabilistic loading: the influence of load correlation", Mechanics Based Design of Structures and Machines, 37(3), pp. 327-348, 2009. https://doi.org/10.1080/15397730902936328

[11] Zhao, J., Wang, C. "Robust topology optimization of structures under loading uncertainty", AIAA Journal, 52(2), pp. 398-407, 2014. https://doi.org/10.2514/1.J052544

[12] Zhao, Q., Chen, X., Ma, Z.-D., Lin, Y. "Robust Topology Optimization Based on Stochastic Collocation Methods under Loading Uncertainties", Mathematical Problems in Engineering, 2015, Article ID: 580980, 2015. https://doi.org/10.1155/2015/580980

[13] Chen, S., Chen, W., Lee, S. "Level set based robust shape and topology optimization under random field uncertainties", Structural and Multidisciplinary Optimization, 41(4), pp. 507-524, 2010. https://doi.org/10.1007/s00158-009-0449-2

[14] Tootkaboni, M., Asadpoure, A., Guest, J. K. "Topology optimization of continuum structures under uncertainty - A Polynomial Chaos approach", Computer Methods in Applied Mechanics and Engineering, 201-204, pp. 263-275, 2012. https://doi.org/10.1016/j.cma.2011.09.009 
[15] Chen, S., Chen, W. "A new level-set based approach to shape and topology optimization under geometric uncertainty", Structural and Multidisciplinary Optimization, 44(1), pp. 1-18, 2011. https://doi.org/10.1007/s00158-011-0660-9

[16] Allaire, G., Dapogny, C. "A linearized approach to worst-case design in parametric and geometric shape optimization", Mathematical Models and Methods in Applied Sciences, 24(11), pp. 2199-2257, 2014. https://doi.org/10.1142/S0218202514500195

[17] Lazarov, B. S., Schevenels, M., Sigmund, O. "Topology optimization considering material and geometric uncertainties using stochastic collocation methods", Structural and Multidisciplinary Optimization, 46(4), pp. 597-612, 2012. https://doi.org/10.1007/s00158-012-0791-7

[18] Zhou, M., Lazarov, B. S., Sigmund, O. "Topology optimization for optical projection lithography with manufacturing uncertainties", Applied Optics, 53(12), pp. 2720-2729, 2014. https://doi.org/10.1364/AO.53.002720

[19] Lazarov, B. S., Schevenels, M., Sigmund, O. "Topology optimization with geometric uncertainties by perturbation techniques", International Journal for Numerical Methods in Engineering, 90(11), pp. 1321-1336, 2012.

https://doi.org/10.1002/nme.3361
[20] Sigmund, O. "Manufacturing tolerant topology optimization", Acta Mechanica Sinica, 25(2), pp. 227-239, 2009. https://doi.org/10.1007/s10409-009-0240-z

[21] Schevenels, M., Lazarov, B. S., Sigmund, O. "Robust topology optimization accounting for spatially varying manufacturing errors", Computer Methods in Applied Mechanics and Engineering, 200(49-52), pp. 3613-3627, 2011.

https://doi.org/10.1016/j.cma.2011.08.006

[22] Keshavarzzadeh, V., Fernandez, F., Tortorelli, D. A. "Topology optimization under uncertainty via non-intrusive polynomial chaos expansion", Computer Methods in Applied Mechanics and Engineering, 318, pp. 120-147, 2017. https://doi.org/10.1016/j.cma.2017.01.019

[23] Bruns, T. E., Tortorelli, D. A. "Topology optimization of non-linear elastic structures and compliant mechanisms", Computer Methods in Applied Mechanics and Engineering, 190(26-27), pp. 3443-3459, 2001. https://doi.org/10.1016/S0045-7825(00)00278-4

[24] MathWorks "MATLAB", (Version 8.5) [computer program] Availaable at: https://uk.mathworks.com [Accessed: 25.01.2019] 